therapy should be influenced by the patient's desire to preserve fertility. At our present state of knowledge, a category of cervical adenocarcinoma that could be treated conservatively in order to preserve fertility cannot be identified.

Patients with IA2 lesions can be treated with primary radical or modified radical hysterectomy or primary radiation therapy with equivalent results. The choice of therapy should be influenced by such factors as ovarian preservation, comorbid conditions, and potential late side effects.

Patients with stages IB and IIA cervical cancer are appropriately treated with either radical hysterectomy with pelvic lymphadenectomy or radiation therapy (external beam therapy and brachytherapy) with equivalent results. The choice of therapy should be influenced by the same factors described in patients with stage IA2 disease. To minimize morbidity, primary therapy should avoid the routine use of both radical surgery and radiation therapy.

The optimal role for imaging studies in defining the extent of disease and in planning radiation therapy needs further investigation as does the measurement of serum tumor markers in patients with invasive cervical cancer.

\section{What is the appropriate management of advanced-stage and recurrent cervical cancer?}

For stage IIB or greater, the standard of care is primary radiation therapy, consisting of external beam radiation using megavoltage radiation energies and brachytherapy. Low-dose-rate brachytherapy (LDR) significantly reduces the rate of local recurrence. The use of highdose rate brachytherapy (HDR) has been increasing, although more studies are needed to define optimal fractionation schemes as well as long-term complications of this method.

Cytotoxic chemotherapy for advanced cervical cancer is currently under study. Cisplatin is the drug with the best documented single-agent activity. At present there is no evidence that the addition of other drugs improves survival. Patients with locally recurrent disease are treat- ed with the modality not previously received. Patients who have had a hysterectomy should receive pelvic radiation therapy. Patients who have had maximal radiation therapy may have surgery, depending on site of recurrence and extent of disease. The clearest role for surgical therapy is for centrally recurrent disease. For most patients, tailored pelvic exenteration remains the standard surgical approach. Aggressive therapies for recurrent disease after radiation are emotionally, physically, and economically costly, and this should be considered in making treatment decisions.

Palliative treatment is appropriate for patients with symptomatic disease and can be achieved in most patients by radiation therapy. Oncologists should assure patients that psychological support and adequate treatment of all symptoms, including pain, are part of the treatment plan.

\section{What are new directions for} research in cervical cancer?

In the area of prevention, more research is needed on the modification of highrisk behavior in young people. In addition, research is needed on the optimal methods of evaluating and treating HIVpositive women with cervical lesions; improved screening in populations that are typically underscreened; provider behaviors that influence patient and clinician compliance with Pap smear screening; and methods of improving the accuracy and interpretation of cytologic sampling techniques, including liquidbased systems and computer automation. Also needed is research to develop markers to predict which women with low-grade lesions are likely to develop high-grade lesions or cancer. Support should also be given to research on top-

(continued on page 266)

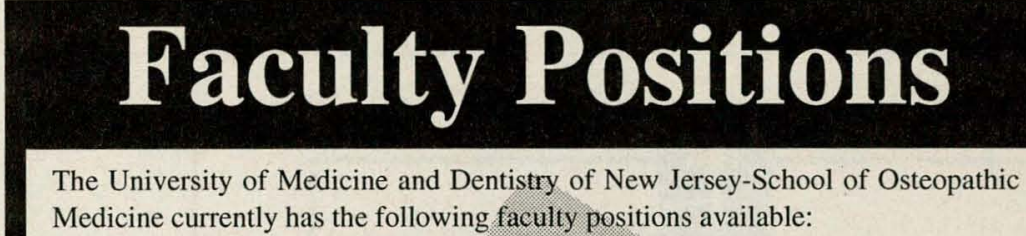 \\ Vice Chairperson Department of Internal Medicine}

Will assist in the administration of the department and will be responsible for planning and developing the department's research program. The successful candidate will be board certified, have a proven track record in research and a background in General Internal Medicine or a medical sub-specialty. You will also be participating in our clinical and academic missions.

\section{General Internists Department of Internal Medicine}

Must be board certified or eligible. Join our osteopathic medical school for professional growth in an academic setting by participating in our clinical, educational and research missions.

We offer a competitive salary and a benefit package that includes malpractice insurance, retirement benefits, paid vacations and holidays as well as health, dental, life and disability insurance. Send C.V., indicating position of interest, to Thomas A. Cavalieri, D.O., Chairperson, Department of Internal Medicine, UMDNJ-

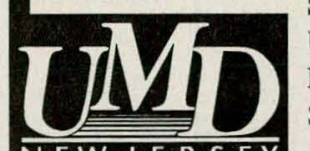
SOM, 42 E. Laurel Road, Suite 3100, Stratford, NJ 08084. UMDNJ is an Affirmative Action/Equal Opportunity Employer, $\mathrm{m} / \mathrm{f} / \mathrm{d} / \mathrm{v}$, and a member of the University Health System of New Jersey. 